\title{
IDENTIFYING TRENDING SENTIMENTS IN THE 2016 U.S. PRESIDENTIAL ELECTION: A CASE STUDY OF TWITTER ANALYTICS
}

\author{
Sri Hari Deep Kolagani, MBA Student, California State University, Chico, skolagani@mail.csuchico.edu \\ Arash Negahban,Ph.D., California State University, Chico, anegahban@csuchico.edu \\ Christine Witt, Ph.D., California State University, Chico, cwitt3@csuchico.edu
}

\begin{abstract}
Social media provides a platform for people to share information, exchange thoughts, and discuss their views about various topics. Sentiment analysis techniques analyze sentiments expressed by people on social media. This study contributes to the emerging research on sentiment analysis of social media content related to a certain event. The goal of this research is to analyze public sentiments associated with the candidates in the United States Presidential Election of 2016. The authors collected more than 200,000 tweets via hashtag for the two major presidential candidates, customized the dictionary based on the political context of the study, and analyzed the tweets in terms of positive and negative as well as eight types of sentiment (anger, anticipation, disgust, fear, joy, sadness, surprise, trust). The result of the study shows significant difference among the candidates in terms of joy, fear, surprise, disgust, trust, while the difference in the rest of the sentiments were not significant. We also tested the difference in the polarity of the sentiments in terms of positive and negative sentiments in general and found that there is a significant difference in positive sentiments between the candidates while the difference in negative sentiments in general was insignificant.
\end{abstract}

Keywords: Sentiment Analysis, Sentiments, Social Media, and Twitter

\section{INTRODUCTION}

In the past decade, a vast amount of data on public opinions has been collected and analyzed. Although more data on public opinions is accessible, determining relevant information from data collected on opinions has proven to be difficult. Sentiment analysis provides an overview of favorable and unfavorable opinions on various topics and subject matter.

Sentiment analysis is sometimes referred to as opinion mining. Sentiment analysis assists researchers in analyzing opinions. Bing (2010) contends sentiment analysis has tremendous value for real-time applications to data collection and analysis. Sentiment analysis provides the edge for analyzing opinions on important events such as political movements. Sentiment analysis can also provide organizations information on their completion, marketing, public relations, and risk management (Wang, Wei, Liu, Zhou, \& Zhang, 2011; Ravi \& Ravi, 2015).

However, the interpretation of opinions can be debatable because determining the emotional tone or conjecture of text has proven to be difficult. Sentiment analysis involves identification of sentiment expressions, polarity, and strength of the expressions and their relationship to the subject. Sentiments are analyzed into categories such as positive or negative or into an $n$-point scale where $n$ represents the number of sentiment category (Prabowo \& Thelwall, 2009).

Sentiment analysis lays the path to the computational study of people's opinions, appraisals, attitudes, and emotions. These opinions can be evaluated toward entities, individuals, issues, events, and topics. Bing and Zhang (2012) found sentiment analysis to be a useful technique despite being technically challenging. A specific challenge exists with developing a deep understanding of syntactical and semantic language rules. It can often be difficult to determine the explicit or implicit, regular and irregular that is needed for effective opinion and sentiment mining (Cambria, Schuller, Xia, \& Havasi, 2013).

Social media has become a substitution of offline media providing a medium for people to participate in political discussions and share political views. Opinions are shared on social media in many forms including textual posts, 
news, images, emoticons, GIF's and videos (Hu \& Huan, 2012). Twitter is a popular social media platform known for massively spreading instant messages called tweets.

Twitter is a microblogging system that allows users to publish tweets of up to 140 characters in length. In the first quarter of 2017, there was an average number of 328 million monthly active Twitter users. Twitter has become a political platform where opinions are presented and exchanged (Agarwal, Xie, Vovsha, Rambow, Passonneau, 2011; Jiang, Yu, Zhou, Liu, \& Zhao, 2011; Cui, Zhang, Liu, \& Ma, 2011). Therefore, Twitter provides real-time access to globally expressed political opinions and sentiments of the 2016 presidential election. The researchers examined the sentiments of tweets that used certain hashtags that identify the presidential nominees Senator Hillary Clinton and Donald Trump.

\section{Twitter as a Medium to Measure Sentiments in Elections}

Researchers have studied the effects of social media on issues in the world's political landscape. In 2012, Sounman \& Nadler completed one of the first empirical studies of social media's potential impact on the U.S. election. Their study examined the 2012 presidential candidates' salience by using the number of mentions of the candidates' names during the election on Twitter. Interestingly the authors found "that while social media does substantially expand the possible modes and methods of election campaigning, high levels of social media activity on the part of presidential candidates have, as of yet, resulted in minimal effects on the amount of public attention they receive online" (p. 455). However, additional studies have found Twitter's impact have contradicted the Sounman \& Nadler (2012) study.

Several researchers have used Twitter in the context of various elections, including in a geolocation-based analysis of the Indian elections (Omaima, et al., 2015) as well as for prediction of electoral results in a multi-party environment of United Kingdom elections 2015 (Burnap, et al., 2016). Previous research has yielded mixed results relating to the correlation between the tweets and the vote share (Bennet, 2016; Jansen \& Koop, 2005). Other researchers have also investigated the usefulness of parts of speech to determine sentiments in the context of microblogging and found that parts-of-speech and emoticons may not be useful for microblogs such as Twitter (Efthymios et al., 2011).

\section{RESEARCH MODEL}

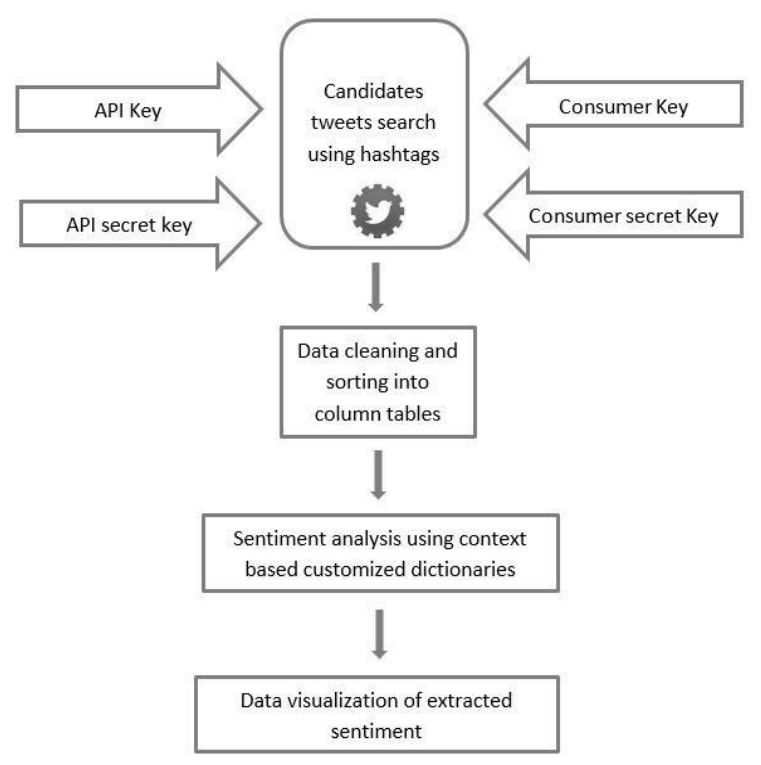

Figure 1. Implemented Research Model for Text Mining, Data Analysis and Visualization 
Data collection from Twitter was initiated using the Twitter Application Programmable Interface (API) that requires an API key, API secret key, consumer key and consumer secret key. This initiation during data collection was achieved with $\mathrm{R}$ and SAP HANA Studio. The data from Twitter was requested using popular hashtags for each candidate. The data collection was completed daily from April 24, 2016 to November 28, 2016. The data contained more than 200,000 tweets including the date of creation and the Tweet ID. Figure 1 depicts the process we used in our text mining and sentiment analysis. First, we collected the Tweets with the hashtags associated with each of the candidates. Then, we cleansed and sorted the data into tables of a columnar database. Finally, we exported the tables as csv files into R and ran the sentiment analysis. The hashtags we used for each of the candidates are shown in Table 1 below.

Table 1. Hashtags Used By Candidate

\begin{tabular}{|l|l|}
\hline Candidate Name & Hashtags Used \\
\hline Donald Trump & \#Trump, \#DonaldTrump, \#Trump2016, \#DonaldTrumpforPresident \\
\hline Hillary Clinton & \#Hillary2016, \#HillaryClinton \\
\hline
\end{tabular}

Dictionaries were used in this algorithm-based sentiment analysis approach to achieve consistency and accuracy. In the case of the 2016 presidential election, there were many positive terms (such as great or stronger) in the candidates' campaign slogans. For this reason, the authors used a context-based custom dictionary by adopting the Stanford CoreNLP, Hu and Lu-KDD-2004 dictionaries. The dictionaries to analyze the tweets were customized to exclude specific words related to the campaigns or slogans, such as the words "trump" and "great." We also added emoticons to the dictionaries to capture the sentiments that the Twitter users expressed via emoticons. TwitteR, Tm, Syuzhet, Ggplot2, Sentiment and stringR packages were used to perceive, process, and present the data dictionaries using R. Once the sentiments were obtained, Tableau was used as a support to visualize the results.

\section{RESULTS}

There was a significant difference in the sentiments of the candidates after the use of a customized context-based dictionary. The outcome, however, did not significantly change though there has been an accuracy enhancement as result of customized dictionary. The results indicate a shift towards the negative axis. Sentiment scores are given to each word within the tweet which are sourced from the pre-described dictionary of positive, negative and neutral words with respective sentiment scores. The value on the sentiment scale is hence the sum of the sentiment scores given to each word within a tweet. Here in our analysis we tried to study the overall sentiments using the aggregated sentiment scores of all the tweets in our dataset. Table 2 below graphs for each candidate reveal the sentiment of each candidate on a scale of -9 to 9 , where -9 being the most negative and +9 being the most positive. 
Table 2. Sentiment analysis of tweets for each candidate before and after dictionary customization

\begin{tabular}{|c|c|c|c|c|}
\hline \multirow[b]{2}{*}{ 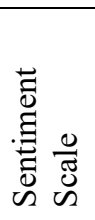 } & \multicolumn{2}{|l|}{ Donald Trump } & \multicolumn{2}{|l|}{ Hillary Clinton } \\
\hline & $\begin{array}{l}\text { Before Dictionary } \\
\text { Customization }\end{array}$ & $\begin{array}{l}\text { After Dictionary } \\
\text { Customization }\end{array}$ & $\begin{array}{l}\text { Before Dictionary } \\
\text { Customization }\end{array}$ & $\begin{array}{l}\text { After Dictionary } \\
\text { Customization }\end{array}$ \\
\hline-9 & 1 & 1 & 1 & 1 \\
\hline-8 & 3 & 3 & 3 & 3 \\
\hline-7 & 10 & 1 & 10 & 10 \\
\hline-6 & 28 & 22 & 30 & 28 \\
\hline-5 & 108 & 87 & 118 & 108 \\
\hline-4 & 434 & 367 & 461 & 434 \\
\hline-3 & 1457 & 1432 & 1578 & 1457 \\
\hline-2 & 4687 & 5527 & 5008 & 4687 \\
\hline-1 & 14034 & 15100 & 14936 & 14034 \\
\hline 0 & 27264 & 29172 & 28603 & 27264 \\
\hline 1 & 12860 & 10852 & 11364 & 12860 \\
\hline 2 & 3575 & 2380 & 2646 & 3575 \\
\hline 3 & 834 & 476 & 539 & 834 \\
\hline 4 & 172 & 66 & 107 & 172 \\
\hline 5 & 26 & 11 & 14 & 26 \\
\hline 6 & 5 & 1 & 1 & 5 \\
\hline 7 & 1 & 0 & 0 & 1 \\
\hline 8 & 0 & 1 & 0 & 0 \\
\hline 9 & 0 & 0 & 0 & 0 \\
\hline
\end{tabular}
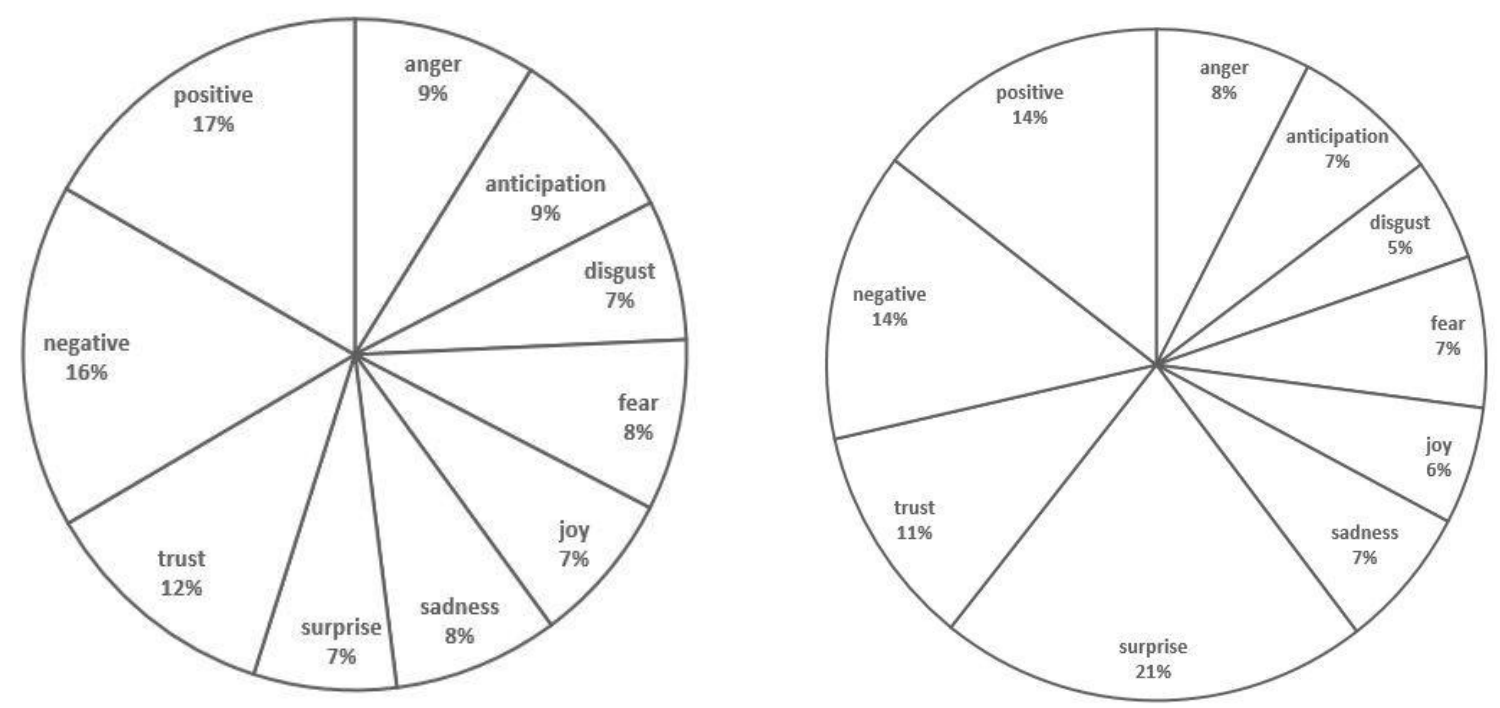

Figure 2. Emotional Analysis of Donald Trump's Tweets Figure 3. Emotional Analysis of Sen. Clinton's Tweets

The above figure illustrates the emotional analysis of tweets related to each candidate. To validate the obtained sentiment analysis results, an Analysis of Variance (ANOVA) was performed using IBM SPSS. The results of the ANOVA suggest a significant difference in terms of disgust, fear, joy, surprise, trust and positive sentiments. Social media especially in this election played a crucial role in terms of exposing the people emotions. While both democrats and republicans had an equal share of fear and disgust towards the opposite candidates, it was interesting to see people expressing joy, surprise and trust carrying emotions. Ill humor, trolls, memes, biased fake news \& polls and many 
other factors can be a reason for these emotions to show significance. All of the remaining differences among the emotions were not significant.

Table 3. ANOVA of extracted emotions

\begin{tabular}{|c|c|c|c|c|c|c|}
\hline \multicolumn{2}{|c|}{ ANOVA Table } & $\begin{array}{ll}\text { Sum } & \text { of } \\
\text { Squares } & \end{array}$ & df & $\begin{array}{l}\text { Mean } \\
\text { Square }\end{array}$ & $\mathbf{F}$ & Sig. \\
\hline \multirow{3}{*}{ anger } & Between Groups & .438 & 1 & .438 & 1.149 & .284 \\
\hline & Within Groups & 49969.319 & 130995 & .381 & & \\
\hline & Total & 49969.757 & 130996 & & & \\
\hline \multirow{3}{*}{ anticipation } & Between Groups & .351 & 1 & .351 & .974 & .324 \\
\hline & Within Groups & 47149.076 & 130995 & .360 & & \\
\hline & Total & 47149.427 & 130996 & & & \\
\hline \multirow{3}{*}{ disgust } & Between Groups & 43.432 & 1 & 43.432 & 151.601 & .000 \\
\hline & Within Groups & 37528.444 & 130995 & .286 & & \\
\hline & Total & 37571.875 & 130996 & & & \\
\hline \multirow{3}{*}{ fear } & Between Groups & 6.740 & 1 & 6.740 & 18.441 & .000 \\
\hline & Within Groups & 47879.066 & 130995 & .366 & & \\
\hline & Total & 47885.806 & 130996 & & & \\
\hline \multirow{3}{*}{ joy } & Between Groups & 23.226 & 1 & 23.226 & 76.946 & .000 \\
\hline & Within Groups & 39540.522 & 130995 & .302 & & \\
\hline & Total & 39563.748 & 130996 & & & \\
\hline \multirow{3}{*}{ sadness } & Between Groups & .301 & 1 & .301 & .886 & .347 \\
\hline & Within Groups & 44571.395 & 130995 & .340 & & \\
\hline & Total & 44571.696 & 130996 & & & \\
\hline \multirow{3}{*}{ surprise } & Between Groups & 17212.335 & 1 & 17212.335 & 54694.364 & .000 \\
\hline & Within Groups & 41224.170 & 130995 & .315 & & \\
\hline & Total & 58436.505 & 130996 & & & \\
\hline \multirow{3}{*}{ trust } & Between Groups & 55.342 & 1 & 55.342 & 108.836 & .000 \\
\hline & Within Groups & 66609.396 & 130995 & .508 & & \\
\hline & Total & 66664.738 & 130996 & & & \\
\hline \multirow{3}{*}{ negative } & Between Groups & .180 & 1 & .180 & .241 & .624 \\
\hline & Within Groups & 98164.329 & 130995 & .749 & & \\
\hline & Total & 98164.510 & 130996 & & & \\
\hline \multirow{3}{*}{ positive } & Between Groups & 10.962 & 1 & 10.962 & 14.978 & .000 \\
\hline & Within Groups & 95868.115 & 130995 & .732 & & \\
\hline & Total & 95879.077 & 130996 & & & \\
\hline
\end{tabular}

These sentiment analyses were compared to the Electoral College and popular vote results of the United States presidential election in 2016. The analysis of the results of the Twitter sentiments favored Senator Hillary Clinton in terms of positive sentiments. It is possible that Twitter users' sentiments may indicate a correlation in how Twitter users plan to vote. A strong positive tweet for a candidate may ultimately result in a vote for that candidate. The results may also indicate that more strong positive tweets for Clinton could correlate with the popular vote outcome. In the 2016 election, Senator Clinton won the popular vote considerably with almost 2.9 million more votes. The strong positive sentiments for Senator Clinton could be influenced by the debates, controversies, interviews, and other significant events.

\section{LIMITATIONS}

Contextualizing the sentiment of tweets is challenging due to the limited contextual information available in a 140character tweet. In addition, the quantity of tweets in this study are only a small sample of the total tweets sent during the timeframe of this study. The sample tweets had additional limitations because a Twitter API was used to collect the tweets. The maximum of tweets allowed to be collected per day is 140,000 . 
Limited search query terms were used to generate the sample tweets. The use of limited hashtags to query the data could have had a considerable effect on the quantity of tweets available for both candidates. This may have resulted in the loss of tweets containing positive or negative sentiments related to both major political party candidates. Demographics along with geographical mapping of the sentiments in the tweets were not considered in this research.

\section{FUTURE RESEARCH}

Data collected from one social media platform may limit the efficiency of the results. Future studies should extend this research to other social media. The tweets were collected randomly without considering factors like demographics or electoral geography. Future studies should examine geographical patterns of contextual information. In addition, further improving the customization of the context-based dictionaries could improve the accuracy of the results. Thus, the area of sentiment analysis has adequate future research to pursue in terms of techniques, data collection, and dictionary customization. To account for the discrepancy of the popular vote and the electoral vote in the 2016 election, researchers could assign categories to determine correlations that are more accurate to the electoral vote.

\section{CONCLUSIONS}

Our results indicate that people use social media platforms such as Twitter to express their positive or negative sentiments. Moreover, these sentiments may extend to the context of the general populations' opinion on the 2016 presidential election events, debates, and controversies. Few studies have been conducted on the efficacy of social media sentiment analysis and the outcomes of major political elections.

\section{REFERENCES}

Agarwal, A., Xie, B., Vovsha, I., Rambow, O., \& Passonneau, R. (2011). Sentiment analysis of twitter data. In Proceedings of the workshop on languages in social media (pp. 30-38). Association for Computational Linguistics.

Bennett, S. (2016). Predicting elections with twitter: What 140 Characters Reveal about Political Sentiment. Retrieved from https://pdfs.semanticscholar.org/2888/d46d7ccfd844d0855dd90155b96ea93540a1.pdf

Bing, L. (2010). Sentiment analysis and subjectivity. Handbook of Natural Language Processing (2nd ed.). Chapman and Hall.

Bing, L., \& Zhang, L. (2012). A survey of opinion mining and sentiment analysis. Mining text data. 415-463

Borondo, J., Morales, A. J., Losada, J. C., \& Benito, R. M. (2012). Characterizing and modeling an electoral campaign in the context of Twitter: 2011 Spanish Presidential election as a case study. Chaos, 22(2), 023138-023138-7. doi:10.1063/1.4729139

Burnap, P., Gibson, R., Sloan, L., Southern, R., \& Williams, M. (2016). 140 characters to victory?: Using Twitter to predict the UK 2015 General Election. Electoral Studies, 41230-233. doi:10.1016/j.electstud.2015.11.017

Cambria, E., Schuller, B., Xia, Y., \& Havasi, C. (2013). New avenues in opinion mining and sentiment analysis. IEEE Intelligent Systems, 28(2), 15-21.

Cui, A., Zhang, M., Liu, Y., \& Ma, S. (2011). Emotion tokens: Bridging the gap among multilingual twitter sentiment analysis. Asia Information Retrieval Symposium (238-249). Springer Berlin Heidelberg.

Efthymios, K., Wilson, T., \& Moore, J. D,. (2011). Twitter sentiment analysis: The good the bad and the omg! Proceedings of the Fifth International AAAI Conference on Weblogs and Social Media. Barcelona, Spain. 


\section{Issues in Information Systems}

Volume 18, Issue 2, pp. 80-86, 2017

Hu, X., \& Huan, L. (2012). Text analytics in social media. In C. C. Aggarwal \& C. Zhai (Eds.), Mining text data (385-414). New York: Springer.

IBM Corp. Released 2013. IBM SPSS Statistics for Windows, Version 22.0. Armonk, NY: IBM Corp.

Jiang, L., Yu, M., Zhou, M., Liu, X., \& Zhao, T. (2011). Target-dependent twitter sentiment classification. In Proceedings of the 49th Annual Meeting of the Association for Computational Linguistics: Human Language Technologies-Volume 1 (pp. 151-160). Association for Computational Linguistics.

Omaima, A., Parack, S., \& Chavan, B. (2015). Application of location-based sentiment analysis using Twitter for identifying trends towards Indian general elections 2014. Proceedings of the 9th International Conference on Ubiquitous Information Management and Communication. ACM, 2015.

Pang, B., \& Lee, L. (2008). Opinion mining and sentiment analysis. Foundations and Trends ${ }^{\circledR}$ in Information Retrieval, 2(1-2), 1-135.

Prabowo, R., \& Thelwall, M. (2009). Sentiment analysis: A combined approach. Journal of Informetrics, 3(2), 143157.

Ravi, K., \& Ravi, V. (2015). A survey on opinion mining and sentiment analysis: Tasks, approaches and applications. Knowledge-Based Systems, 8914-8946. doi:10.1016/j.knosys.2015.06.015 .

Sounman, H., \& Nadler, D. (2012). Which candidates do the public discuss online in an election campaign?: The use of social media by 2012 presidential candidates and its impact on candidate salience. Government Information Quarterly, 29(4), 455-461. doi:10.1016/j.giq.2012.06.004.

R Core Team (2013). R: A language and environment for statistical computing. R Foundation for Statistical Computing, Vienna, Austria. URL http://www.R-project.org/.

Wang, X., Wei, F., Liu, X., Zhou, M., Zhang, M. (2011). Proceedings of the 20th ACM International conference on Information and knowledge management. Glasgow, Scotland. 